\title{
Boundary layer analysis and heat transfer of a nanofluid
}

\author{
M.M. MacDevette • T.G. Myers • B. \\ Wetton
}

Received: date / Accepted: date

\begin{abstract}
A theoretical model for nanofluid flow, including Brownian motion and thermophoresis, is developed and analysed. Standard boundary layer theory is used to evaluate the heat transfer coefficient near a flat surface. The model is almost identical to previous models for nanofluid flow which have predicted an increase in the heat transfer with increasing particle concentration. In contrast our work shows a marked decrease indicating that under the assumptions of the model (and similar ones) nanofluids do not enhance heat transfer. It is proposed that the discrepancy between our results and previous ones is due to a loose definition of the heat transfer coefficient and various ad hoc assumptions.
\end{abstract}

Keywords Nanofluid · Convective heat transfer · Boundary layer · Heat transfer coefficient

\section{Introduction}

There exists a vast literature regarding the behaviour and applications of nanofluids. In particular the often remarkable experimental results concerning

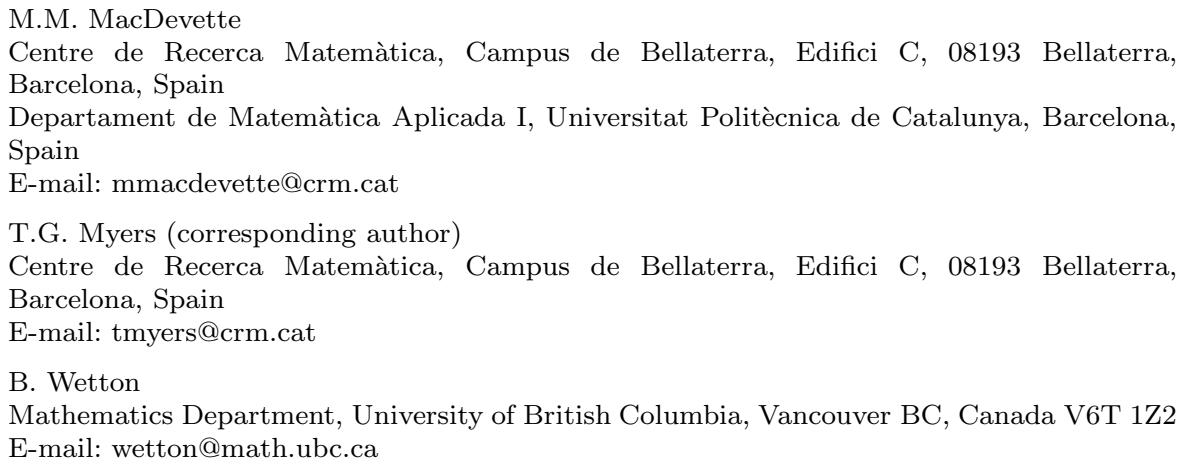


their heat transfer properties has seen them proposed as a front runner in the race to cool modern high performance electronic equipment. However, there appears no real consensus on whether nanofluids are indeed capable of removing large amounts of heat. The plethora of experimental papers promoting their efficiency and enhanced thermal conductivity, see (Haddad et al. 2012; Hwang et al. 2012; Kleinstreuer and Feng 2011) for example, appeared to have been superseded by the benchmark study (Buongiorno et al. 2009) carried out in over 30 organisations throughout the world which suggested no anomalous enhancement of thermal conductivity in the fluids tested. Indeed this should not be too surprising since heat conduction occurs due to the transfer of kinetic energy from hot, rapidly vibrating atoms or molecules to their cooler, more slowly vibrating neighbours. In solids the close, fixed arrangement of atoms means that conduction is more efficient than in fluids, which have a larger distance between atoms (Myers et al. 2012). A nanofluid is made up of a small quantity of solid particles separated by a large amount of fluid, thus ruling out the possibility of a great deal of intimate contact between particles and hence suggesting no significant increase in thermal conductivity. This conclusion is backed up by a small number of other theoretical and experimental papers showing a degradation in thermal performance with increasing volume fraction (Haddad et al. 2012; Popa et al. 2011; Yang et al. 2005). Yet despite this conclusion nanofluids and their heat transfer properties are still the subject of numerous articles, as discussed in the recent review of Kleinstreuer and Feng (2011).

In addition to the lack of consensus on physical properties there is not yet an accepted model for nanofluid flow. A number of models and physical mechanisms (which may or may not be important) are described by Das et al. (2003). In this paper we will focus on a particular form of model, originally proposed by Buongiorno (2006), which includes thermophoresis and Brownian motion. In the paper by Buongiorno (2006) an order of magnitude study was carried out to dismiss a number of heat transfer mechanisms and show that thermophoresis and Brownian motion play a significant role in the energy transport of a flowing liquid. He then analysed a boundary layer flow model to demonstrate an increase in heat transfer coefficient with particle volume fraction. Subsequently various theoretical papers based on the same model have verified this conclusion (Jang and Choi 2006; Kuznetsov and Nield 2010; Maiga et al. 2004; Xuan and Roetzel 2000). Evans et al. (2006) concluded that Brownian motion has a negligible effect on the thermal conductivity. Savino and Paterna (2008) study buoyancy driven flow in a $1 \mathrm{~mm}$ wide channel, they conclude that Brownian motion and thermophoresis do affect the flow, but only over a time-scale of 27 hours, with the results most noticeable when gravity is $10^{-6}$ of its normal value. The real goal in the development of nanofluids for cooling purposes is to enhance the heat transfer and energy removal from a given surface. To understand this requires knowledge of the flow and heat transfer coefficient (HTC) at the interface between the fluid and the solid. This is the aim of the present paper. The HTC is a surprisingly poorly defined quantity so, in the following section, we will begin by examining the HTC and 
defining it in a way that reflects the correct heat transfer from a surface. We will then develop a similar model to that of Buongiorno (2006), Savino and Paterna (2008) via the energy and momentum equations defined in chapter 3 of Bird et al. (2007). Standard boundary layer scaling will be applied to reduce the equations and, in particular, demonstrate that Brownian motion and thermophoresis are negligible within the boundary layer. We go on to show that, for the fluid-particle systems investigated, the HTC decreases with increasing particle volume fraction. Finally, given the number of papers that have reached the opposite solution from the same equations we briefly discuss reasons for this discrepancy. Throughout the paper we will work with a waterbased nanofluid, although parameter values and some results are also reported for Ethyene Glycol based nanofluids.

\section{Calculating the heat transfer coefficient}

The goal of this paper is to determine whether the addition of nanoparticles to a base fluid can improve its ability to transfer heat. It is important to bear in mind that this heat transfer depends not only on the heat removal from the surface but also how well the fluid transports the energy away. For example, although the nanofluid may have a higher heat capacity than the base fluid, this increased ability to store thermal energy may be offset by the increase in fluid viscosity, meaning energy transport with the flow is slower. Hence, in assessing the fluid's heat removal ability we must consider the coupled problem of heat and fluid flow. A second significant issue in analysing the heat removal is the concept of the heat transfer coefficient. This is the parameter used by many authors to quantify the heat transfer from a solid to a fluid. However, it is loosely defined and often does not truly reflect the amount of heat transferred to the fluid. Consequently, before we move on to the full thermal model we will begin with a discussion of the HTC.

When fluid flows over a solid surface the HTC represents the ratio of heat input at the boundary to that transferred to the fluid. If $Q$ is the energy input at the boundary per unit area and $\Delta T$ some temperature change in the fluid then the HTC is typically defined by $h=Q / \Delta T$. If the no-slip condition holds at the solid-liquid boundary then the heat transfer there is by conduction, rather than convection, and so Fourier's law holds,

$$
Q=-\left.k \frac{\partial T}{\partial y}\right|_{y=0},
$$

where $y=0$ denotes the position of the interface. Substituting for $Q$ leads to the standard boundary condition

$$
-\left.k \frac{\partial T}{\partial y}\right|_{y=0}=h \Delta T .
$$

The variation in interpretation of this boundary condition comes through the choice of $\Delta T$. Perhaps the most common choice is $\Delta T=T_{w}-T_{\infty}$, where 
$T_{w}$ is the temperature of the solid and $T_{\infty}$ that of the fluid in the far field (Bejan 2004; Das et al. 2003). If the solid is heating the fluid, then it is wellknown that $T_{w}>T_{y=0^{+}}$and so, since $h \propto 1 / \Delta T$, the definition $\Delta T=T_{w}-T_{\infty}$ will underestimate the HTC. The mathematical literature tends to favour the choice $\Delta T=T_{y=0^{+}}-T_{\infty}$, which should be closer to representing the heat passed to the fluid. In fact the two choices are often combined by choosing a mathematical description where the fluid temperature $T_{y=0^{+}}=T_{w}$. In the nanofluid literature such choices may be found in (Buongiorno 2006; Daungthongsuk and Wongwises 2007; Popa et al. 2011; Xuan and Roetzel 2000) for example and there are also numerous examples in the general heat transfer literature (Bird et al. 2007).

In fact, neither of the above options actually represents the energy increase in the fluid. The problem being that the choice of $\Delta T$ is arbitrary and implicitly assumes that the temperature (or energy) rise is linear in the fluid. To correctly determine the energy rise requires knowledge of the velocity and temperature profiles in the liquid. Say a fluid enters a system at $x=0$ with some initial temperature $T_{\infty}$ and a corresponding energy flux then a distance $L$ downstream the energy flux above the initial value is given by

$$
\int_{0}^{\delta_{T}(L)} \rho c u\left(T-T_{\infty}\right) d y
$$

where $\delta_{T}(L)$ is the thickness of the thermal boundary layer at $x=L$. In order to write a HTC in a manner similar to previous definitions we may define an average temperature rise in the fluid $T_{a v}$ by

$$
\left(T_{a v}-T_{\infty}\right) \int_{0}^{\delta_{T}} \rho c u d y=\int_{0}^{\delta_{T}} \rho c u\left(T-T_{\infty}\right) d y .
$$

Note, $T_{a v}$ is referred to in Bird et al. (2007) as the 'cup' average. The term cup average indicates that if the flow to the edge of the boundary layer at $x=L$ was collected in a cup the temperature of this fluid would be given by $T_{a v}$. The HTC that correctly reflects the ratio of energy entering at the boundary to that transferred into the fluid is

$$
h=\frac{Q}{T_{a v}-T_{\infty}}=\frac{Q \int_{0}^{\delta_{T}} \rho c u d y}{\int_{0}^{\delta_{T}} \rho c u\left(T-T_{\infty}\right) d y} .
$$

This is the definition we will use in the following work. Note, the HTC varies with distance downstream (we have omitted writing $x=L$ in all the integrals) but will tend to an asymptote far downstream. A more detailed description of the HTC and different ways to estimate it are given in Bird et al. (2007).

\section{Mathematical Modelling}

Initially we will assume fluid properties, such as density, viscosity, thermal conductivity and heat capacity, depend on the volume fraction $\phi$. Hence we 
write down a general model to account for this. The equations governing the flow of a compressible nanofluid may be written as

$$
\begin{aligned}
\frac{\partial \rho_{n f}}{\partial t}+\nabla \cdot\left(\rho_{n f} \mathbf{u}\right) & =0 \\
\rho_{n f}\left[\frac{\partial \mathbf{u}}{\partial t}+\mathbf{u} \cdot \nabla \mathbf{u}\right] & =-\nabla p-\nabla \cdot \tilde{\tau}+\rho_{n f} \mathbf{g}, \\
\frac{\partial\left(\chi_{n f} T\right)}{\partial t}+\nabla \cdot\left(\chi_{n f} \mathbf{u} T\right) & =\nabla \cdot\left(k_{n f} \nabla T\right)+\mu_{n f} \Phi, \\
\frac{\partial \phi}{\partial t}+\nabla \cdot(\phi \mathbf{u}) & =\nabla \cdot\left[D_{B} \nabla \phi+D_{T} \frac{\nabla T}{T}\right],
\end{aligned}
$$

where $\mathbf{u}$ is the velocity vector, $T$ the temperature, $\phi$ the volume fraction of nanoparticles and $g$ gravity. Subscripts $b f, n f$ and $n p$ refer to the base fluid, nanofluid and nanoparticle, respectively. The density, volumetric heat capacity and viscous dissipation are defined as

$$
\begin{aligned}
& \rho_{n f}=\phi \rho_{n p}+(1-\phi) \rho_{b f}, \\
& \chi_{n f}=(\rho c)_{n f}=\phi \rho_{n p} c_{n p}+(1-\phi) \rho_{b f} c_{b f} \\
& \Phi=\frac{\partial u_{i}}{\partial x_{j}}\left(\frac{\partial u_{i}}{\partial x_{j}}+\frac{\partial u_{j}}{\partial x_{i}}\right) .
\end{aligned}
$$

The derivation of equations (6-8) follows along the lines described in Bird et al. (2007), Ch. 3. Although it should be noted that in the derivation of Bird et al. (2007) the specific heat capacity is assumed constant. In that case combining the energy and continuity equations permits $\chi$ to be moved outside the derivative terms on the left hand side of (8). However, since the specific heat of a nanofluid is a function of $\phi$ for the moment we leave the equations in a slightly more general form.

Brownian diffusion is represented by the term involving $D_{B}$ in equation (9), the $D_{T}$ term describes thermophoresis. The velocity induced by a temperature gradient is typically written as

$$
v=-\beta \mu_{b f} \nabla T /\left(\rho_{b f} T\right),
$$

where $\beta=0.26 \cdot k_{b f} /\left(2 k_{b f}+k_{n p}\right)$ is a proportionality factor between that of the slip velocity due to thermophoresis and the temperature factor $\nabla T / T$, see (Astumian 2007; Brenner and Bielenberg 2005; Duhr and Braun 2006; McNab and Meisen 1973; Savino and Paterna 2008). The thermophoretic mass flux is then $j_{T}=\rho_{n p} \phi v$. This is often written as $j_{T}=-\rho_{n p} \tilde{D}_{T} \nabla T$ where $\tilde{D}_{T}=\beta \mu_{b f} \phi /\left(\rho_{b f} T\right)$ is termed the thermal diffusion coefficient. However, this 'diffusion coefficient' has dimensions $\mathrm{m}^{2} /(\mathrm{s} \mathrm{K})$. Consequently in equation (9) we follow the convention of Buongiorno (2006) in defining a dimensionally correct diffusion coefficient, $D_{T}=\beta \mu_{b f} \phi / \rho_{b f}$, which then requires an additional factor $1 / T$ in the final term of equation (9). Vigolo et al. (2010) also point out this discrepancy in definition and term $\tilde{D}_{T}$ as used by many previous authors as a thermophoretic mobility rather than a diffusion coefficient. The two 
diffusion coefficients, $D_{B}, D_{T}$, involve the variable temperature and volume fraction respectively,

$$
D_{B}=\frac{k_{B} T}{3 \pi \mu_{b f} d_{p}}, \quad D_{T}=\frac{\beta \mu_{b f} \phi}{\rho_{b f}},
$$

where $k_{B}$ is the Boltzmann constant and $d_{p}$ the particle diameter. Previous studies have evaluated $D_{B}, D_{T}$ using some reference temperature and volume fraction, whilst allowing $T, \phi$ to vary everywhere else in the equations, see for example (Buongiorno 2006; Savino and Paterna 2008). To clarify the dependence on temperature and volume fraction in all subsequent equations we will use constants that do not involve $T$, $\phi$, i.e. $C_{B}=\frac{D_{B}}{T}, C_{T}=\frac{D_{T}}{\phi}$. Note, we write $C$ 's instead of $D$ 's to clarify that these are not the standard diffusion coefficients (and indeed $C_{B}$ no longer has the correct dimensions for this).

The components of the stress tensor are given in various co-ordinate systems in [(Bird et al. 2007), Ch. 3]. In Cartesian co-ordinates

$$
\begin{aligned}
& \tilde{\tau}_{x x}=\mu_{n f}\left[-2 \frac{\partial u}{\partial x}+\frac{2}{3} \nabla \cdot \mathbf{u}\right], \tilde{\tau}_{x y}=\tilde{\tau}_{y x}=-\mu_{n f}\left(\frac{\partial u}{\partial y}+\frac{\partial v}{\partial x}\right), \\
& \tilde{\tau}_{x z}=\tilde{\tau}_{z x}=-\mu_{n f}\left(\frac{\partial u}{\partial z}+\frac{\partial v}{\partial y}\right),
\end{aligned}
$$

and the components $\tilde{\tau}_{y y}, \tilde{\tau}_{z z}$ follow the pattern of $\tilde{\tau}_{x x}$. The viscosity, $\mu_{n f}$, may vary considerably with the particle volume fraction. The Brinkman relation for the viscosity significantly under predicts the true value for $\phi>0.01$. Khanafer and Vafai (2011) present a complex polynomial representation for $\mu_{n f}=\mu_{n f}(\phi, T)$ and show good agreement with data. The experiments of Prasher et al. (2006) indicate only a weak dependence of viscosity on $T$ and particle diameter. Corcione (2011) shows a weak dependence on $T$ but presents evidence for the diameter dependence. In the absence of agreement we will begin with a simple representation, where $\mu_{n f}=\mu_{n f}(\phi)$, given by Maiga et al. (2004) who fitted the experimental data of a water based nanofluid by Wang et al. (1999) with the following relation

$$
\mu_{n f}=\left(1+7.3 \phi+123 \phi^{2}\right) \mu_{b f},
$$

and for ethylene glycol based fluids,

$$
\mu_{n f}=\left(1-0.19 \phi+306 \phi^{2}\right) \mu_{b f} .
$$

The thermal conductivity of a nanofluid is a thorny issue, with much discrepancy and debate concerning the often remarkable experimental results. The basic theoretical model, the Maxwell model, provides a simple relation for $k_{n f}$ in terms of $\phi$ and $k_{b f}$. For $\phi \ll 1$ this relation may be linearised to show

$$
k_{n f}=k_{b f}\left(1+C_{k} \phi\right),
$$

where $C_{k} \approx 3$. This is well-known to significantly underpredict the thermal conductivity. The Maxwell model is based on a steady-state analysis: a recent 
theory involves determining the conductivity via a time-dependent analysis which leads to $C_{k} \approx 5$ and provides significantly improved agreement with published data (Myers et al. 2013). The new equation that takes into account the volume fraction and particle properties is given by

$$
k_{n f}=\frac{k_{b f}}{\left(1-\phi^{1 / 3}\right)^{2}}\left[(1-\phi)+\phi \frac{\rho_{n p} c_{n p}}{\rho_{b f} c_{b f}}\right] \frac{n-1}{2(n+1)}\left[\frac{1+\phi^{1 / 3}}{2}-\frac{1}{n+1}\right]^{-1}
$$

where $n=2.233$ is a constant determined by the boundary condition (Myers et al. 2013).

To reduce the complexity of the system we now make the following assumptions:

1. Viscous dissipation is negligible, $\Phi \approx 0$. This will be the case for most standard nanofluid flows and is clear from the numerical solutions provided in the paper by Koo and Kleinstreuer (2005) for flow with $U \approx 2 \mathrm{~m} / \mathrm{s}$ in a channel of around $50 \mu \mathrm{m}$. Their results for water and $\mathrm{CuO}$ nanoparticles showed a negligible difference with and without viscous dissipation, while excluding the dissipation in ethylene glycol led to a very slight difference.

2. Gravity is negligible (this is simply so we may focus on the heat transfer, rather than the driving force).

3. The system is in a steady state, so we are considering the fully-developed region.

Under these assumptions the flow is now governed by

$$
\begin{aligned}
\nabla \cdot\left(\rho_{n f} \mathbf{u}\right) & =0 \\
\rho_{n f} \mathbf{u} \cdot \nabla \mathbf{u} & =-\nabla p+\nabla \cdot(\tilde{\tau}) \\
\nabla \cdot\left(\chi_{n f} \mathbf{u} T\right) & =\nabla \cdot\left(k_{n f} \nabla T\right) \\
\nabla \cdot(\phi \mathbf{u}) & =\nabla \cdot\left[C_{B} T \nabla \phi+C_{T} \phi \frac{\nabla T}{T}\right] .
\end{aligned}
$$

In the following section we will analyse this system subject to a uniform flux condition along a flat boundary, $y=0$,

$$
k_{n f} T_{y}=-Q .
$$

The flow is subject to no-slip conditions at $y=0$

$$
u=v=0,
$$

while the inlet values, at $x=0$, are defined as

$$
\phi=\phi_{\text {in }} \quad T=T_{\infty} \quad \mathbf{u}=(U, 0) .
$$

The above system is almost identical to that of Buongiorno (2006). To obtain that system requires assuming incompressible flow (so reducing equation (20) to $\nabla \cdot \mathbf{u}=0)$, setting $C_{B}=D_{B} / T, C_{T}=D_{T} / \phi$ in (23) gives the steady, incompressible version of Buongiorno (2006), eq. 17, the energy equation Buongiorno 
(2006), eq. 23 differs from our equation (22) since it includes thermophoresis and Brownian motion. However, the scaling later shows this to be negligible (in the paper by Buongiorno (2006) the Lewis number, of order $10^{5}$, divides both terms) and in fact both terms are neglected in all subsequent analysis. Savino and Paterna (2008) work in terms of mass fraction rather than $\phi$. Their system is also similar to ours, but they retain the time dependence and include gravity in the momentum equation to permit buoyancy effects.

In the following section we carry out the standard boundary layer analysis on equations (20-23) to determine the fluids ability to remove heat. This requires us to augment the boundary conditions (24-26) with the far-field conditions

$$
u=U \quad v=0 \quad T=T_{\infty} \quad \text { as } \quad y \rightarrow \infty .
$$

\section{Steady-state boundary layer analysis}

The standard boundary layer scaling (Acheson 1990) has

$$
\begin{aligned}
& \hat{x}=\frac{x}{L} \quad \hat{y}=\frac{y}{L} \sqrt{R e} \quad \hat{T}=\frac{T-T_{\infty}}{A} \\
& \hat{u}=\frac{u}{U} \quad \hat{v}=\frac{v}{U} \sqrt{R e} \quad \hat{p}=\frac{p-p_{\infty}}{\rho_{b f} U^{2}},
\end{aligned}
$$

where $U$ is the flow velocity in the far field (equal to the inlet velocity), $R e=\rho_{b f} U L / \mu_{b f}$ is the Reynolds number and $A$ is an as yet undetermined temperature scale. An important point to note here is that we take $U$ as a fixed velocity scale, that is, it does not vary with $\phi$. Using scalings independent of particle load allows us to directly compare results in subsequent sections but, since an increase in $\phi$ will lead to an increased viscosity, this means that fixed $U$ requires an increase in pressure gradient. In the following section we will show that the heat transfer decreases with increasing $\phi$, however since $U$ is fixed our result actually shows the HTC in a better light than it deserves. In fact, not only does the HTC decrease with increasing $\phi$ with our model but it also costs more energy to move the fluid.

The variable physical parameters are scaled with the base fluid value

$$
\hat{\mu}_{n f}=\frac{\mu_{n f}}{\mu_{b f}} \quad \hat{\rho}_{n f}=\frac{\rho_{n f}}{\rho_{b f}} \quad \hat{k}_{n f}=\frac{k_{n f}}{k_{b f}} \quad \hat{\phi}=\frac{\phi}{\phi_{i n}} \quad \hat{\chi}_{n f}=\frac{\chi_{n f}}{\chi_{b f}} .(30)
$$

At present this scaling will lead to a form of boundary layer system. To simplify the problem we first examine the effect of the scaling on the $\phi$ equation (23). Dropping the hat notation this becomes

$$
\nabla \cdot(\phi \mathbf{u})=\gamma \frac{\partial}{\partial y}\left[\left(T+\frac{T_{\infty}}{A}\right) \frac{\partial \phi}{\partial y}+\lambda \frac{\phi}{T+T_{\infty} / A} \frac{\partial T}{\partial y}\right]
$$

where $\gamma=C_{B} A \rho_{b f} / \mu_{b f}, \lambda=C_{T} /\left(C_{B} A\right)$. The temperature scale is chosen based on the input of heat to the system, so we non-dimensionalize the 
boundary condition in equation (24), $\frac{k_{b f} A \sqrt{R e}}{L} T_{y}=-Q$, and choose $A=$ $Q L /\left(k_{b f} \sqrt{R e}\right)$. In Table 1 we present the device and material properties for ethylene glycol (EG) and water based nanofluids with $\mathrm{Al}_{2} \mathrm{O}_{3}$ particles, from which the values for the coefficients in the non-dimensional equations are calculated: these are presented in Table 2. For EG $\gamma=\mathcal{O}\left(10^{-5}\right)$ and water $\gamma=\mathcal{O}\left(10^{-4}\right) \ll 1$ and so the right hand side of $(31)$ is negligible. Consequently we may write

$$
\nabla \cdot(\phi \mathbf{u})=\phi \nabla \cdot(\mathbf{u})+\mathbf{u} \cdot \nabla \phi \approx 0
$$

The steady-state continuity equation, equation (20), with the definition of $\rho_{n f}$ given by equation (10), expands to

$$
\left(\phi \rho_{n p}+(1-\phi) \rho_{b f}\right) \nabla \cdot \mathbf{u}+\left(\rho_{n p}-\rho_{b f}\right) \mathbf{u} \cdot \nabla \phi=0
$$

From equation (32) we may substitute $\mathbf{u} \cdot \nabla \phi=-\phi \nabla \cdot \mathbf{u}$ which then allows all $\phi$ terms to be cancelled and leads to $\nabla \cdot \mathbf{u}=0$, that is, the fluid is incompressible. We may then write

$$
\mathbf{u} \cdot \nabla \phi=0
$$

The physical significance of this equation is that $\phi$ is constant along the streamlines. From the inlet condition we know the non-dimensional volume fraction $\phi=1$ and so on all streamlines $\phi=1$ (hence $\phi=1$ everywhere). Since $\gamma=\mathcal{O}\left(10^{-4}\right)$ for water the approximation $\phi=1$ is accurate to $\mathcal{O}\left(10^{-2}\right) \%$, and for ethylene glycol it is even more accurate. Put another way, since the diffusion effects due to Brownian motion and thermophoresis are so small the particles simply move with the fluid and, in particular, are not affected by the heat input at the boundary. Although the value of $\gamma$ may vary with the nanofluid or heat flux, its miniscule value indicates particle diffusion through Brownian motion or thermophoresis is unlikely to ever play an important role in the boundary layer flow of a nanofluid. This concurs with the findings of Evans et al. (2006), who used molecular dynamics to demonstrate that Brownian motion only has a minor effect on the enhancement of thermal conductivity of nanofluids.

The conclusion that $\phi=1$ has significant implications for the mathematical model, for example it shows that physical quantities such as $\rho_{n f}, \mu_{n f}$ and $k_{n f}$ are also constant and determined via the non-dimensional versions of (10), (19) and $(16,17)$. With constant physical quantities we may apply standard boundary layer theory to the $u$ and $T$ equations. 
Table 1 Device and material properties of water and ethylene glycol (EG) based nanofluids with $\mathrm{Al}_{2} \mathrm{O}_{3}$ nanoparticles. (Data taken from Buongiorno (2006) and Wikipedia).

\begin{tabular}{llll} 
Quantity & Symbol & Units & Value \\
\hline Particle diameter & $d_{p}$ & $m$ & $20 \times 10^{-9}$ \\
Far-field temperature & $T_{\infty}$ & $K$ & 300 \\
Plate flux & $Q$ & $W m^{-2}$ & $10^{7}$ \\
Length of plate & $L$ & $\mathrm{~m}$ & $10^{-2}$ \\
Far-field velocity & $U$ & $\mathrm{~ms}^{-1}$ & $10^{-1}$ \\
Thermal conductivity $\left(\mathrm{Al}_{2} \mathrm{O}_{3}, \mathrm{EG}, \mathrm{H}_{2} \mathrm{O}\right)$ & $k_{n p}, k_{b f}$ & $\mathrm{Wm}^{-1} \mathrm{~K}^{-1}$ & $30,0.258,0.609$ \\
Density $\left(\mathrm{Al}_{2} \mathrm{O}_{3}, \mathrm{EG}, \mathrm{H}_{2} \mathrm{O}\right)$ & $\rho_{n p}, \rho_{b f}$ & $\mathrm{kgm}^{-3}$ & $3950,1108.8,10^{3}$ \\
Specific heat capacity $\left(\mathrm{Al}_{2} \mathrm{O}_{3}, \mathrm{EG}, \mathrm{H}_{2} \mathrm{O}\right)$ & $c_{n p}, c_{b f}$ & $\mathrm{Jg}^{-1} \mathrm{~K}^{-1}$ & $800,2360,4187$ \\
Viscosity (EG, $\left.\mathrm{H}_{2} \mathrm{O}\right)$ & $\mu_{b f}$ & $\mathrm{Nsm}^{-2}$ & $1.61 \times 10^{-2}, 10^{-3}$ \\
Boltzmann's constant & $k_{B}$ & $\mathrm{JK}^{-1}$ & $1.38 \times 10^{-23}$
\end{tabular}

Table 2 Values of coefficients in non-dimensional equations based on properties given in Table 1.

\begin{tabular}{lll} 
Quantity & ethylene glycol & Water \\
\hline$C_{B}$ & $4.3825 \times 10^{-15}$ & $7.0559 \times 10^{-14}$ \\
$C_{T}$ & $3.1918 \times 10^{-8}$ & $5.0721 \times 10^{-9}$ \\
$\beta$ & 0.0022 & 0.0051 \\
$\operatorname{Re}$ & 68.8696 & $10^{3}$ \\
$\operatorname{Pr}$ & 147.2713 & 6.8752 \\
$A$ & $4.6705 \times 10^{4}$ & $5.1926 \times 10^{3}$ \\
$\gamma$ & $1.2714 \times 10^{-8}$ & $3.6638 \times 10^{-7}$ \\
$\lambda$ & 155.9362 & 13.8437
\end{tabular}

With the above scaling the governing steady-state equations become

$$
\begin{aligned}
\frac{\partial u}{\partial x}+\frac{\partial v}{\partial y} & =0 \\
\rho_{i}\left(u \frac{\partial u}{\partial x}+v \frac{\partial u}{\partial y}\right) & =-\frac{\partial p}{\partial x}+\mu_{i} \frac{\partial^{2} u}{\partial y^{2}}+\mathcal{O}(1 / R e) \\
0 & =\frac{\partial p}{\partial y}+\mathcal{O}(1 / R e) \\
u \frac{\partial T}{\partial x}+v \frac{\partial T}{\partial y} & =\frac{k_{i}}{\chi_{i}} \frac{1}{\operatorname{Pr}} \frac{\partial^{2} T}{\partial y^{2}}+\mathcal{O}(1 / R e),
\end{aligned}
$$

where the subscript $i$ denotes the inlet value of each quantity for the nanofluid and the Prandtl number $\operatorname{Pr}=\mu_{b f} \chi_{b f} /\left(\rho_{b f} k_{b f}\right)$. Equation (37) indicates $p=$ $p(x)$. In keeping with standard boundary layer theory we note that approaching the far-field, $y \rightarrow \infty$, then $u \rightarrow 1, v \rightarrow 0$ and so equation (36) determines $p_{x}=0$. Hence the problem is now described by (35) and

$$
\begin{gathered}
u \frac{\partial u}{\partial x}+v \frac{\partial u}{\partial y}=\nu_{i} \frac{\partial^{2} u}{\partial y^{2}} \\
u \frac{\partial T}{\partial x}+v \frac{\partial T}{\partial y}=\frac{k_{i}}{\chi_{i}} \frac{1}{P r} \frac{\partial^{2} T}{\partial y^{2}},
\end{gathered}
$$


where $\nu_{i}=\mu_{i} / \rho_{i}$. These equations and the subsequent boundary conditions could be further simplified by choosing the inlet values of the physical parameters in the scaling but this would mean that the length and height scales would vary for each inlet volume fraction. Using the base fluid values means that our subsequent results may be compared on the same graph, with the same scales.

The imposed non-dimensional boundary conditions are

$$
\begin{aligned}
& u=v=0, \quad k_{i} T_{y}=-1 \quad \text { at } \quad y=0, \\
& u=1, v=0, T=0 \quad \text { as } \quad y \rightarrow \infty .
\end{aligned}
$$

\subsection{Flow over a flat plate}

The well-known Blasius solution for boundary layer flow over a flat plate (see Acheson (1990) for example) involves first introducing a stream function $\psi$ where

$$
u=\frac{\partial \psi}{\partial y} \quad v=-\frac{\partial \psi}{\partial x} .
$$

This automatically satisfies the continuity equation, (35). The similarity variable $\eta=y / \sqrt{2 \nu_{i} x}$ is then introduced. To satisfy the momentum equation, (39), requires $\psi=\sqrt{2 \nu_{i} x} f(\eta)$ where $f$ is an unknown function determined from

$$
f^{\prime \prime \prime}+f f^{\prime \prime}=0,
$$

where primes denote differentiation with respect to $\eta$. This is simply the transformed version of equation (39). The boundary conditions $u=v=0$ at $y=0$ and $u \rightarrow 1$ as $y \rightarrow \infty$ become

$$
f(0)=f^{\prime}(0)=0 \quad f^{\prime}(\infty)=1 .
$$

In fact this boundary value problem may be simplified using Töpfer's transformation $f(\eta)=r F(r \eta)$, where $r>0$ is a constant. The Blasius equation remains the same

$$
F^{\prime \prime \prime}+F F^{\prime \prime}=0
$$

but it may be solved subject to the conditions

$$
F(0)=0 \quad F^{\prime}(0)=0 \quad F^{\prime \prime}(0)=1,
$$

that is, we solve an initial value problem (which is much easier to deal with than a boundary value problem). The solutions of the two systems will coincide provided

$$
f^{\prime}(\infty)=r^{2} F^{\prime}(\infty)=1
$$

which then requires $r=\left(F^{\prime}(\infty)\right)^{-1 / 2}$. The numerical solution of $(46,47)$ determines $r \approx 0.7773$.

The same transformation could be used to reduce the heat equation but the flux condition at $y=0$ does not permit a similarity form. Consequently, at this stage we must look for an approximate solution form. 
4.2 Solution by Heat Balance Integral Method (HBIM)

A standard approximate method to analyse boundary layer flow was developed by von Karman and Pohlhausen. From this stemmed the Heat Balance Integral Method (HBIM) which is primarily used on thermal problems. Obviously we are interested in the boundary layer flow, but there has been much more research on the HBIM so we will use techniques developed for this method to analyse the current problem. Specifically, we will employ a technique described in (Myers $2010 \mathrm{~b}$ ) to approximate the momentum and thermal boundary layer flow of a power law fluid.

If we consider the flow equation, equation (39), then the HBIM involves choosing a simple function to approximate the velocity over a finite boundary layer $\delta(x)$. For $y>\delta$ the deviation of velocity from that of the bulk flow is negligible (although we do not yet define what constitutes negligible). In this case the velocity boundary layer would be defined by the boundary conditions $u(x, \delta)=1$ and $u_{y}(x, \delta)=0$. If the approximating function is a polynomial of the form

$$
u=a_{0}+a_{1}\left(1-\frac{y}{\delta}\right)+a_{p}\left(1-\frac{y}{\delta}\right)^{p},
$$

then the conditions $u(x, \delta)=1$ and $u_{y}(x, \delta)=0$ determine $a_{0}=1, a_{1}=0$. The no-slip condition at $y=0$ determines $a_{p}=-1$ and so

$$
u=1-\left(1-\frac{y}{\delta}\right)^{p} .
$$

The expression for $u$ involves two unknowns, $\delta(x)$ and $p$. The velocity boundary layer thickness is determined by integrating equation (39) over the region $y \in[0, \delta]$

$$
\int_{0}^{\delta} u u_{x}+v u_{y} d y=\nu_{i} \int_{0}^{\delta} u_{y y} d y .
$$

Noting from the continuity equation (35) $u_{x}=-v_{y}$, we may write the above integral momentum equation

$$
\frac{d}{d x} \int_{0}^{\delta} u(1-u) d y=\left.\nu_{i} \frac{\partial u}{\partial y}\right|_{y=0},
$$

see (Chhabra and Richardson 2008; Myers 2010 b) for more details. Substituting for $u$ from equation (50) leads to a single ordinary differential equation for $\delta$ with solution

$$
\delta=\alpha \sqrt{x}
$$

where $\alpha=\sqrt{2 \nu_{i}(p+1)(1+2 p)}$ and $\delta(0)=0$. The standard HBIM and the von Karman-Pohlhausen technique take $p=2$ however a more accurate method, developed by Myers $(2009 ; 2010$ a) is to choose $p$ to minimise the least-squares error when the approximating function is substituted back into the momentum equation. In this case the error function is defined as

$$
E_{p}=\int_{0}^{\delta}\left(\frac{\partial G}{\partial x}-\nu_{i} \frac{\partial^{2} u}{\partial y^{2}}\right)^{2} d y
$$


where $G=u(u-1)$. Note that $\nu_{i}$ is a function of the inlet values, and so depends on the inlet volume fraction. Consequently the optimal $p$ value could also vary with the volume fraction, however our calculations with $\phi_{\text {in }}$ varying between $1 \%$ and $10 \%$ show $p=2.7237$ with less than a $10^{-2} \%$ variation. To demonstrate the result of this method in Figure 1 we compare velocity profiles of (50) with $p=2.7237$ and that of the Blasius solution at $x=0.1$ with $\phi_{\text {in }}=5 \%$. Near the surface, $y=0$, the agreement is excellent. For $y>1$ there is some divergence, but the solutions both end close to $y=2.5$ (indicating the $\delta$ calculation is accurate). However, for practical purposes the important point is that the approximation works well near the boundary, so allowing an accurate calculation of the drag coefficient. The decay to the far field velocity is of less practical importance. In (Myers 2010 b) the similarity solution for a Newtonian fluid with a fixed temperature boundary condition was also calculated and compared with the HBIM solution. Again the approximation was excellent near the boundary (in fact better than the velocity boundary layer). In this case it allowed the HTC to be accurately calculated.

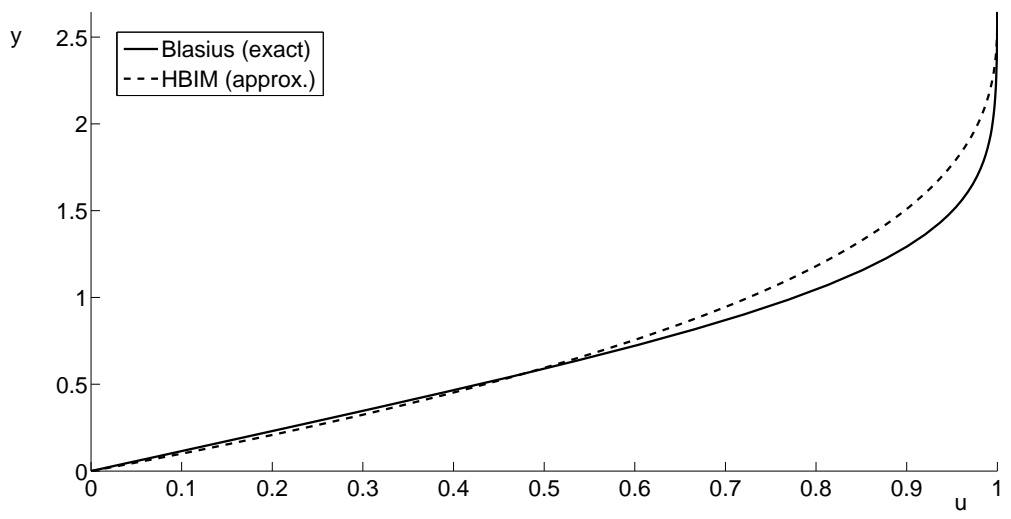

Fig. 1 Comparison of the exact Blasius and approximate HBIM solutions for the velocity profile at $x=0.1$ with $\phi_{\text {in }}=5 \%$.

For the energy equation we define $\delta_{T}$ as the thickness of the thermal boundary layer, then the conditions $T\left(x, \delta_{T}\right)=0, T_{y}\left(x, \delta_{T}\right)=0, k_{i} T_{y}(x, 0)=-1$ determine the temperature profile

$$
T=\frac{\delta_{T}}{k_{i} q}\left(1-\frac{y}{\delta_{T}}\right)^{q}
$$

The heat balance integral is determined by integrating the energy equation (40) over $y \in\left[0, \delta_{T}\right]$

$$
\frac{d}{d x} \int_{0}^{\delta_{T}} u T d y=-\left.m_{i} \frac{\partial T}{\partial y}\right|_{y=0}=\frac{m_{i}}{k_{i}}
$$


where $m_{i}=k_{i} /\left(\chi_{i} \operatorname{Pr}\right)$, see (Myers $\left.2010 \mathrm{~b}\right)$ for details.

Substituting for $u, T$ from $(50,55)$ requires numerical integration since $p$ is a non-integer, however an analytical solution is preferred so, following Chhabra and Richardson (2008), Myers (2010), we assume that the thermal boundary layer is much smaller than the velocity boundary layer

$$
\delta_{T}=\epsilon \delta
$$

where $\epsilon \ll 1$ (this is confirmed a priori) is to be determined. On applying this to (50) and noting that within the HBI $y \ll \delta_{T}$ we can make the approximation

$$
u \approx 1-\left(1-\frac{p y}{\delta}\right)=\frac{p y}{\delta} .
$$

Now the HBI (56) may be integrated analytically giving

$$
\epsilon=\sqrt[3]{\frac{m_{i} q(q+2)(q+1)}{p \alpha^{2}}} .
$$

The exponent $q$ is found by minimising the error

$$
E_{q}=\int_{0}^{\delta_{T}}\left(\frac{\partial F}{\partial x}-m_{i} \frac{\partial^{2} T}{\partial y^{2}}\right)^{2} d y
$$

where $F=u T$. According to equation (60) the value of $q$ may vary with $m_{i}$, which depends on $k_{i}, \chi_{i}$ and $\operatorname{Pr}$, so it may be a function of material properties and volume fraction. For water, with $\phi_{i n} \in[1,10] \%$ we find $q=1.9975$ (with less than $0.01 \%$ variation), for EG $q=2.014$ (with less than $0.4 \%$ variation). We then find that for $\phi_{i n} \in[1,10] \%$ the value of the small parameter varies $\epsilon \in[0.2971,0.2635]$.

With no analytical solution to verify the thermal approximation we developed a finite difference code to solve equations $(35,39,40)$ subject to boundary conditions $(41,42)$ and inlet conditions $u=1, v=0$ and $T=0$. The semi-infinite $y$ range was cut off at large $y$ where conditions (42) applied. For example at $x=0.1$ it was sufficient to calculate results to $y=5$. Standard finite difference approximations were made for the derivatives in $y$ and the solution was marched in $x$ with implicit stepping. The results showed reasonable agreement with the temperature profiles predicted by equation (55) with the worst disagreement occurring at $y=0$. With $x=0.1$ the HBIM and numerical solutions differed by approximately $10 \%$. We will discuss this difference later with regard to Figure 4.

In Figure 2 we plot velocity and thermal boundary layers for three different volume fractions, $\phi_{i n}=1,5,10 \%$ which are represented by dotted, dashed and solid lines respectively. First we note that $\delta_{T} \ll \delta$ in all cases, confirming the assumption made earlier. A more important point is that the thermal boundary layer thickness increases with volume fraction suggesting that the nanofluid could indeed extract more heat with higher nanoparticle concentrations. In Figure 3 we show the velocity profile $u(y)$ at $x=0.05$ for the same three 


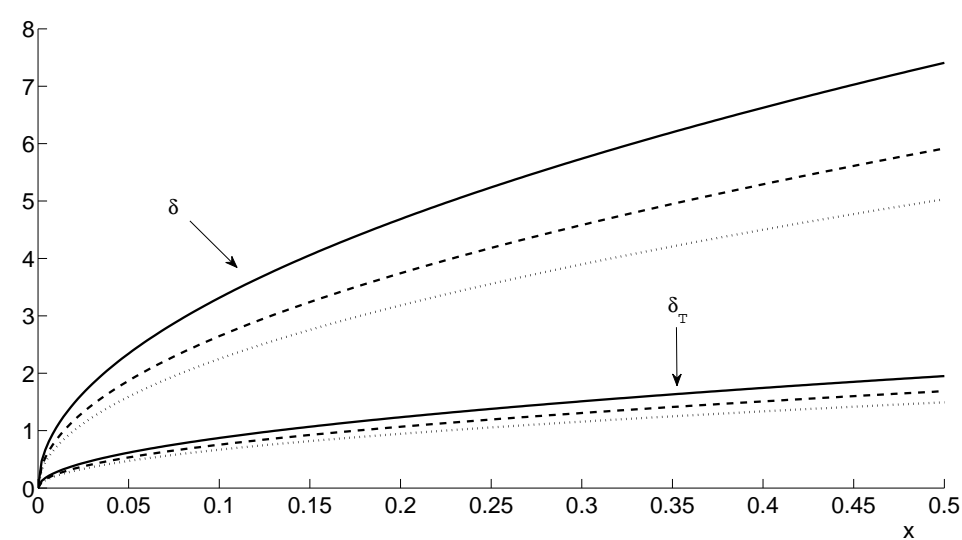

Fig. 2 Delta profiles are compared for different volume fractions $\phi_{i n}=1,5,10 \%$ which are represented by dotted, dashed and solid lines, respectively, for an $\mathrm{Al}_{2} \mathrm{O}_{3}$-water nanofluid.

volume fractions. Here we note that velocity decreases with increasing volume fraction, indicating that the mass transport is slower, which may then have an adverse effect on heat transport.

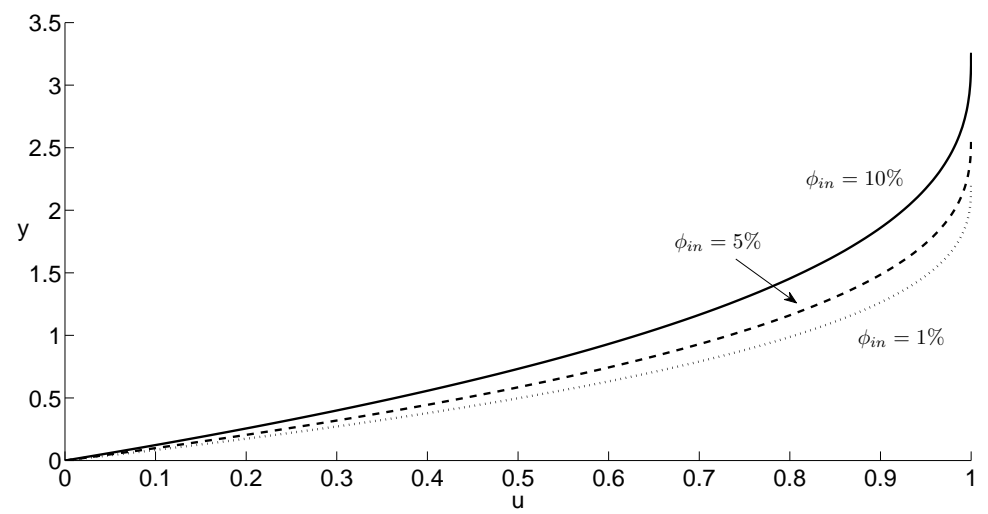

Fig. 3 Velocity profiles predicted by the HBIM for different volume fractions $\phi_{\text {in }}=1,5,10 \%$ which are represented by dotted, dashed and solid lines, respectively, for an $\mathrm{Al}_{2} \mathrm{O}_{3}$-water nanofluid.

Figures 2 and 3 show the trade off between increasing the heat flow (through increasing $k$ ) whilst decreasing the fluid flow (through increasing $\mu$ ) by adding nanoparticles. To determine which effect is dominant we now return to the HTC calculation. Defining a non-dimensional HTC $\hat{h}=L h /\left(k_{b f} \sqrt{R e}\right)$ and 
dropping the hat notation, equation (5) may be written

$$
h_{n f}=\frac{\int_{0}^{\delta_{T}} u d y}{\int_{0}^{\delta_{T}} u T d y} .
$$

In Figure 4 the HTC (61) is plotted against the device length for three values of the volume fraction, $\phi_{i n}=1,5,10 \%$, and the base fluid. What is clear from this graph is that the HTC decreases with increasing volume fraction. Furthermore, as discussed earlier the velocity scale $U$ is fixed in all cases, and so an increase in $\phi_{i n}$ (which increases viscosity) requires an increase in $\Delta p$. So with higher particle loadings the system requires more energy to move the fluid and this further decreases the system's efficiency. That is, according to the present mathematical model and for the cases we have studied for fluid and heat flow of a nanofluid there is no augmentation in the HTC; in fact it appears that the opposite occurs, despite the numerous claims based on the same theory. However, our theoretical result does concur with Ding et al. (2007) who state that nanofluids with an enhanced thermal conductivity do not guarantee an enhancement in the convective heat transfer. Li and Peterson (2010) present experimental results showing a decrease in HTC during natural convection of $\mathrm{Al}_{2} \mathrm{O}_{3}$-water nanofluids: a similar deterioration for $\mathrm{Al}_{2} \mathrm{O}_{3}, \mathrm{CuO}$ and $\mathrm{TiO}_{2}$-water nanofluids is shown by Putra et al. (2003).

Earlier we discussed the error in using the HBIM approximation to the temperature. At $x=0.1$ we found an error of approximately $10 \%$ between the approximate and numerical solutions. From Figure 4 we see that there is an approximately $30 \%$ decrease in HTC, which cannot be explained away by a $10 \%$ error in temperature. In the following section we will discuss why so many researchers have found the opposite result to ours using a similar initial set of equations.

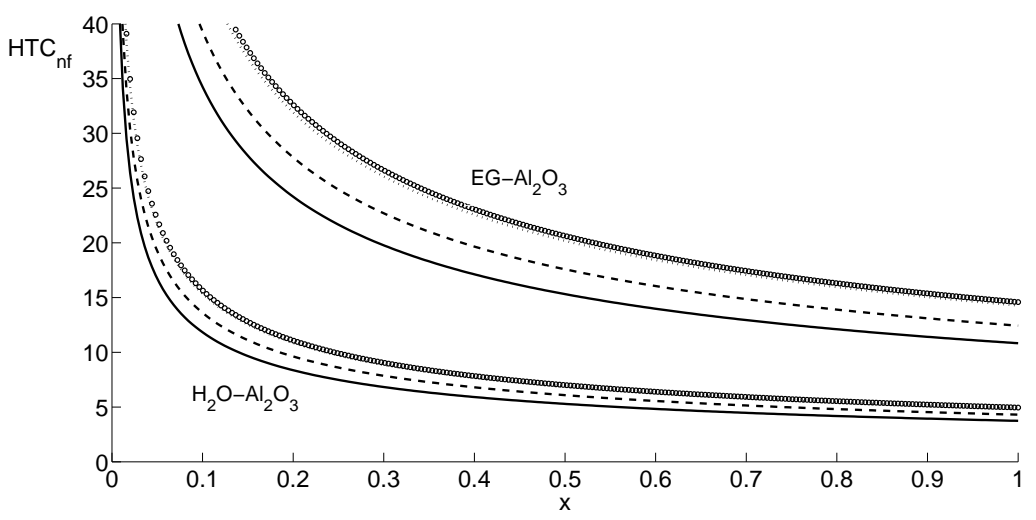

Fig. 4 Variation of the heat transfer coefficient along the surface for the base fluid (circles), ethylene glycol or water, and $\phi_{i n}=1,5,10 \%$ represented by dotted, dashed and solid lines respectively. 


\section{Apparent improvement in HTC?}

Buongiorno (2006) wrote the seminal paper describing the mathematical model for nanofluid heat transfer including thermophoresis and Brownian motion. Savino and Patterson (2008) developed a similar model accounting for gravity effects. Numerous theoretical studies have been based on the form of model developed in these two papers and, in contrast to the present conclusion, the authors generally conclude that the HTC increases with volume fraction. This raises the obvious question of why do our results differ from this previous body of work? There exist too many studies to go through each one individually, so now we will focus on a few highly cited ones.

Buongiorno's model is a special case of that described in the present paper. In $\S 5$ of his paper he investigates the HTC in a 'laminar sublayer' where the flow is described by

$$
\frac{\partial}{\partial y}\left(\mu_{n f} \frac{\partial v}{\partial y}\right)=0 \quad \frac{\partial}{\partial y}\left(k_{n f} \frac{\partial T}{\partial y}\right)=0 \quad \frac{\partial}{\partial y}\left(D_{B} \frac{\partial \phi}{\partial y}+\frac{D_{T}}{T} \frac{\partial T}{\partial y}\right)=0 .
$$

This region is matched to a turbulent sublayer. Equations (62) are formally derived from equations (21-23) by rescaling height to focus on a region close to the wall. To reproduce this we alter the scaling given by equations $(28,29)$ to $y=H \bar{y}, v=H U \bar{v} / L$, where $H$ is an as yet unspecified height-scale (previously we chose $H=L / \sqrt{R e}$ ). Scaling equation (23) leads to

$$
\nabla \cdot(\phi \mathbf{u})=\frac{C_{B} A L}{H^{2} U} \frac{\partial}{\partial y}\left[\left(T+\frac{T_{\infty}}{A}\right) \frac{\partial \phi}{\partial y}+\frac{\lambda \phi}{T+T_{\infty} / A} \frac{\partial T}{\partial y}\right] .
$$

In the analysis of the previous sections we neglected the right hand side of this equation since the leading coefficient was small. To retrieve the third equation in (62) we require this same coefficient to be large, which indicates

$$
H^{2} \ll \frac{C_{B} A L}{U} \Rightarrow H \ll \frac{C_{B} Q L}{k_{b f} U},
$$

after applying the definition $A=Q H / k_{b f}$. To get some idea of the thickness $H$ of this sublayer consider water flowing at a velocity $10 \mathrm{~cm} / \mathrm{s}$ over a section with $L=1 \mathrm{~cm}$ and $C_{B}=7 \times 10^{-14}$, as shown in Tables 1 and 2 . This results in $H \ll 100 \mathrm{~nm}$. It is well known from experiments that the actual laminar sublayer has a thickness $\delta \approx 11.6 \mu /(\rho U)$ : taking the base fluid values for the viscosity and density from Table 1 gives $\delta \approx 0.1 \mathrm{~mm}$. This indicates that for water the system of equations (62) is valid in a region three orders of magnitude smaller than the actual laminar sublayer thickness, and so cannot be matched to the outer turbulent region. In fact this conclusion is independent of the velocity scale since for water, using the parameter values given in Tables 1 and $2, H / \delta=C_{B} Q L \rho_{b f} /\left(11.6 k_{b f} \mu_{b f}\right) \approx 10^{-3}$. For ethylene glycol the ratio is even smaller $H / \delta=10^{-5}$ and the equations hold in a region five orders of magnitude smaller than the width of the laminar sublayer. 
Our analysis in the previous section used boundary layer theory. This is a standard technique to determine flow and heat transfer at a boundary. The key to boundary layer theory is finding an appropriate similarity variable which transforms the governing partial differential equations to ordinary differential form. Kuznetsov and Nield (2010) add buoyancy to Buongiorno's system and seek similarity solutions involving the variable $\eta \propto y / x^{1 / 4}$. Their governing equation for $\phi$ then reduces to an ordinary differential equation,

$$
g^{\prime \prime}+\frac{3}{4} L_{e} \psi g^{\prime}+\frac{N_{t}}{N_{b}} \theta^{\prime \prime}=0
$$

where $g, \theta$ are the functions representing the non-dimensional $\phi, T$ and $\psi$ is the stream function. The strength of Brownian motion and thermophoresis are represented by the coefficients $N_{b}, N_{t}$ respectively and $L_{e}=\alpha_{n f} / D_{B}$ is the Lewis number. Values for these coefficients are calculated by Buongiorno (2006) (with $N_{b} / N_{t}$ denoted $N_{B T}$ ). The values quoted for water containing $10 \mathrm{~nm}$ alumina nanoparticles are $L_{e}=8 \times 10^{5}, N_{B T}=0.2$; for $10 \mathrm{~nm}$ copper nanoparticles $L_{e}=7 \times 10^{5}, N_{B T}=2$. The extremely high value of $L e$ indicates that the non-dimensional equation (65) is incorrectly scaled. Dividing through by $L_{e}$ leads to $g^{\prime} \approx 0$ and consequently $\phi$ is constant (as determined in our analysis). However, the results presented by Kuznetsov and Nield (2010) show a significant variation in $g$, which then affects the velocity and temperature profiles (since $g$ appears in all three governing equations) and consequently affects the heat transfer. The reason for this difference between our conclusion of constant $\phi$ and theirs is that they choose $L_{e}=10$, rather than the correct physical value. The larger value would make the boundary layer thickness negligible. However, the negligible terms in (65) could be retained by introducing a new height scale (of the order $1 / L_{e}$ smaller than the boundary layer heightscale) but again this could not be matched to an outer turbulent region and so their boundary conditions of constant $T, \phi$ in the far-field do not hold.

Khan and Pop (2010) use a slightly different scaling to Kuznetsov and Nield (2010) for the similarity variable to obtain an equation almost identical to (65). Their Lewis number is defined as $L_{e}=\nu / D_{B}$, where $\nu$ is the kinematic viscosity. For water $\nu \approx 10^{-6}$ and so $L_{e}=\mathcal{O}\left(5 \times 10^{4}\right)$. They also employ the value $L_{e}=10$.

Savino and Paterna (2008) study a system almost identical to our initial system (6-9). The focus of their study is convective motion of a nanofluid contained between two differentially heated plates held $1 \mathrm{~mm}$ apart and they do determine a difference in motion due to the effects of thermophoresis and Brownian motion. However, their time-scale is of the order $10^{5} \mathrm{~s}$ or 27 hours for motion in a $1 \mathrm{~mm}$ gap and the results are most noticeable in conditions where gravity is $10^{-6}$ of the standard value.

\section{Conclusion}

In this paper we have developed a model for nanofluid flow including the effects of thermophoresis and Brownian motion, with the aim of determining 
whether nanofluids improve heat removal. The heat transfer coefficient was examined within standard boundary layer theory. The boundary layer formulation showed quite clearly that, close to the substrate thermophoresis and Brownian motion effects are negligible compared to advection. Consequently the particle volume fraction remains constant along the streamlines and so the fluid properties, such as density, viscosity and specific heat, are also constant.

The most significant result of this analysis is the clear decrease in the heat transfer coefficient as the particle concentration increases. From the governing equations this is not an obvious conclusion, since the addition of nanoparticles changes the thermal properties and viscosity of the fluid. Nanoparticles increase the viscosity, density and conductivity however they may decrease the product $\rho c$ (with water a $10 \%$ increase in $\mathrm{Al}_{2} \mathrm{O}_{3}$ nanoparticles decreases $\rho c$ by around $2.5 \%$ ). Hence, there is a trade-off between the enhanced properties, the increased viscosity and possible decrease in $\rho c$. In fact our negative result was somewhat flattering with regards to the HTC since we used a fixed farfield velocity. This means that as the particle concentration increases, and so the viscosity also increases, the pressure drop or pumping power must also be increased. So, not only did the HTC decrease with particle concentration but it cost more energy to pump the fluid.

Our result contradicts many analyses based on similar governing equations, however, it is backed up by recent experimental evidence, see (Buongiorno et al. 2009; Ding et al. 2007; Li and Peterson 2010; Putra et al. 2003) for example. Our model also required a number of assumptions and simplifications, but these could all be quantified and are not of sufficient magnitude to alter our conclusion regarding the heat transfer. Possible reasons for this discrepancy between our conclusion and that of previous papers include

- the rather loose definition of heat transfer coefficient prevalent in the literature

- a number of unjustified assumptions and approximations to reduce the governing equations to a simpler form

- incorrect parameter values, which then enhance the thermophoresis and Brownian motion effects.

This paper does not prove that nanofluids cannot improve heat removal. It may be that the enhancement observed in some experiments is due to a physical effect not included in our model. Further, we have only presented results for water or ethylene glycol based fluids with $\mathrm{Al}_{2} \mathrm{O}_{3}$ particles (although results with $\mathrm{CuO}$ particles were found to be similar) in a single flow configuration. It is possible that some other fluid-solid combinations may have a beneficial effect on heat transfer. However, given the clear deterioration in HTC shown by our calculations, there would need to be a marked change in the fluid properties (such as a much larger increase in thermal conductivity coupled with a much lower viscosity increase) for the current form of model to suggest that nanofluids can improve heat removal significantly. 


\section{Acknowledgements}

MMM gratefully acknowledges the support of a $\mathrm{PhD}$ grant from the Centre de Recerca Matemática. TM gratefully acknowledges the support of this research through the Marie Curie International Reintegration Grant Industrial applications of moving boundary problems Grant no. FP7-256417 and Ministerio de Ciencia e Innovación Grant MTM2011-23789. BW also received funding from the Marie Curie International Reintegration Grant. TM acknowledges the kind support of Prof. J.B. van den Berg and the Mathematics Department of the Vrije Universiteit of Amsterdam, where this work was completed.

\section{References}

1. Acheson DJ (1990) Elementary Fluid Dynamics. Oxford University Press.

2. Astumian RD (2007) Coupled transport at the nanoscale: The unreasonable effectiveness of equilibrium theory. Proceedings of the National Academy of Sciences 104(1):3-4.

3. Bejan A (2004) Convection Heat Transfer (3rd Ed.) John Wiley and Sons.

4. Bird R, Stewart W, Lightfoot E (2007) Transport phenomena. John Wiley and Sons.

5. Buongiorno J (2006) Convective Transport in Nanofluids. Journal of Heat Transfer $128: 240-250$

6. Buongiorno J, Venerus D, Prabhat N, McKrell T, Townsend J et al. (2009) A benchmark study on the thermal conductivity of nanofluids. Journal of Applied Physics 106, 094312.

7. Brenner H, Bielenberg JR (2005) A continuum approach to phoretic motions: Thermophoresis. Physica A 355: 251273.

8. Chhabra RP, Richardson JF (2008) Non-Newtonian flow and applied rheology (2nd Ed.) Butterworth-Heinemann

9. Corcione M (2011) Empirical correlating equations for predicting the effective thermal conductivity and dynamic viscosity of nanofluids. Energy Conversion and Management 52:789793

10. Das S, Putra N, Thiesen P, Roetzel W (2003) Temperature Dependence of Thermal Conductivity Enhancement for Nanofluids. Journal of Heat Transfer 125:567574.

11. Daungthongsuk W, Wongwises S (2007) A critical review of convective heat transfer of nanofluids. Renewable and Sustainable Energy Reviews 11: 797817

12. Ding Y, Chen H, He Y, Lapkin A, Yeganeh A, Siller L and Butenko YV (2007) Forced convective heat transfer of nanofluids. Advanced Powder Technology 18:813824.

13. Duhr S, Braun D (2006) Why molecules move along a temperature gradient. Proceedings of the National Academy of Sciences 103(52): 19678 - 19682.

14. Evans W, Fish J, Keblinski P (2006) Role of Brownian motion hydrodynamics on nanofluid thermal conductivity. Applied Physics Letters 88, 093116.

15. Haddad Z, Abu-Nada E, Oztop HF, Mataoui A (2012) Natural convection in nanofluids: Are the thermophoresis and Brownian motion effects significant in nanofluid heat transfer enhancement? International Journal of Thermal Sciences 57:152-162.

16. Hwang KS, Jang SP, Choi SUS (2009) Flow and convective heat transfer characteristics of water-based $\mathrm{Al} 2 \mathrm{O} 3$ nanofluids in fully developed laminar flow regime. International Journal of Heat and Mass Transfer 52:193-199.

17. Jang SP, Choi SUS (2006) Cooling performance of a microchannel heat sink with nanofluids. Applied Thermal Engineering 26(1718):24572463.

18. Khan WA, Pop I (2010) Boundary-layer flow of a nanofluid past a stretching sheet. International Journal of Heat and Mass Transfer 53: 24772483.

19. Khanafer K., Vafai K. A critical synthesis of thermophysical characteristics of nanofluids. International Journal of Heat and Mass Transfer 54 (2011) 44104428.

20. Kleinstreuer C, Feng Y Experimental and theoretical studies of nanofluid thermal conductivity enhancement: a review. Nanoscale Research Letters 2011, 6:229. 
21. Koo J, Kleinstreuer C (2005) Laminar nanofluid flow in microheat-sinks. International Journal of Heat and Mass Transfer 48:26522661.

22. Kuznetsov AV, Nield DA (2010) Natural convective boundary-layer flow of a nanofluid past a vertical plate International Journal of Thermal Sciences 49:243247.

23. Lee J, Mudawar I (2007) Assessment of the effectiveness of nanofluids for single-phase and two-phase heat transfer in micro-channels. International Journal of Heat and Mass Transfer 50(34):452463.

24. Li CH, Peterson GP (2010) Experimental Studies of Natural Convection Heat Transfer of Al2O3/DI Water Nanoparticle Suspensions (Nanofluids). Advances in Mechanical Engineering 2010 Article ID 742739.

25. Maiga SEB, Nguyen CT, Galanis N, Roy G (2004) Heat transfer behaviors of nanofluids in a uniformly heated tube. Superlattices and Microstructures 35:543-557.

26. McNab GS, Meisen A (1973) Thermophoresis in Liquids. Journal of Colloid and Interface Science 44(2).

27. Myers TG (2009) Optimizing the exponent in the heat balance and refined integral methods, International Communications in Heat and Mass Transfer 36 (2): 143-147.

28. Myers TG (2010 a) Optimal exponent heat balance and refined integral methods applied to Stefan problems, International Journal of Heat and Mass Transfer 53: 1119-1127.

29. Myers TG (2010 b) An approximate solution method for boundary layer flow of a power law fluid over a flat plate. International Journal of Heat and Mass Transfer 53: 23372346.

30. Myers TG, Mitchell SL and Font F (2012) Energy conservation in the one-phase supercooled Stefan problem, International Communications in Heat and Mass Transfer 39, 15221525.

31. Myers TG, MacDevette MM, Ribera H (2013) A time dependent model to determine the thermal conductivity of a nanofluid. Journal of Nanoparticle Research 15:1775.

32. Popa C, Polidori G, Arfaoui A, Fohanno S (2011) Heat and Mass Transfer in External Boundary Layer Flows Using Nanofluids. Heat and Mass Transfer - Modeling and Simulation doi: 10.5772/1431.

33. Prasher R, Bhattacharya P, Phelan PE (2006) Brownian-Motion-Based ConvectiveConductive Model or the Effective Thermal Conductivity of Nanofluids. Journal of Heat Transfer 128:588-595.

34. Putra N, Roetzel W, Das SK (2003) Natural convection of nano-fluids. Heat and Mass Transfer 39(8-9), 775784.

35. Savino R, Paterna D (2008) Thermodiffusion in nanofluids under different gravity conditions. Physics of Fluids 20, 017101.

36. Travis KP, Todd BD, Evans DJ (1997) Departure from Navier-Stokes hydrodynamics in confined liquids. Physical Review E 55(4).

37. Vigolo D, Rusconi R, Stone HA, Piazza R (2010) Thermophoresis: microfluidics characterization and separation. Soft Matter 6:3489-3493.

38. Wang X, Xu X, Choi SUS (1999) Thermal conductivity of nanoparticle-fluid mixture. Journal of Thermophysics and Heat Transfer 13:474-480.

39. Xuan Y, Li Q (2000) Heat transfer enhancement of nanofluids. International Journal of Heat and Fluid Flow 21(1):58-64.

40. Xuan Y, Roetzel W (2000) Conceptions for heat transfer correlation of nanofluids. International Journal of Heat and Mass Transfer 43(19):37013707.

41. Yang Y, Zhong ZG, Grulke EA, Anderson WB, Wu G (2005) Heat transfer properties of nanoparticle-in-fluid dispersion (nanofluids) in laminar flow. International Journal of Heat and Mass Transfer 48:1107-1116. 